\title{
Intraepithelial lymphocytes in the bovine uterus during the oestrous cycle and early gestation
}

\author{
A. L. Vander Wielen and G. J. King \\ Department of Animal \& Poultry Science, University of Guelph, Guelph, Ontario, Canada N1G 2WI
}

\begin{abstract}
Summary. The numbers of lymphocytes occurring in $5 \mathrm{~mm}$ of uterine epithelium were counted in cyclic heifers on Days 5, 10, 15 and 20 after oestrus and in pregnant heifers between Days 19 and 27 after insemination. Specimens were taken from the oviducal, middle and body regions of the ipsilateral and contralateral uterine horns of the pregnant and cyclic animals. Mean \pm s.e.m. lymphocyte numbers in cyclic animals were $25.8 \pm 1.5$ on Day $5,19.7 \pm 5.5$ on Day $10,22.0 \pm 0.6$ on Day 15 and $28.2 \pm 1.5$ on Day 20 after oestrus. There was a significant reduction in lymphocyte numbers as the chorion and uterine epithelium developed into a functional placenta from $24.5 \pm$ 1.1 on Day 19 to $9.0 \pm 0.9$ on Day 27. Qualitative observations suggested an increase in degenerating cells concomitant with the decrease in lymphocytes. Some of these degenerating cells may have been lymphocytes while others were probably uterine epithelial cells. As gestation progressed and the placenta was established, the number of potentially damaging cells at the fetal-maternal interface was reduced.
\end{abstract}

\section{Introduction}

Bovine pregnancy is a parabiotic coexistence between a fetus and dam with the chorion forming an uninterrupted boundary in intimate contact with maternal tissue. It might be anticipated that expression of paternal antigens by the conceptus could render fetal tissue vulnerable to immunological rejection. Since this does not usually occur, the conceptus must be afforded special immunological exemption. Cursory observations suggested a reduction in the number of lymphocytes in the uterine epithelium during the period when the bovine placenta is forming (Wille \& Leiser, 1977; King, Atkinson \& Robertson, 1981, 1982) but quantitative results have not been reported. It was believed that one possible mechanism for altering the maternal immunological status could operate through reduction in numbers of lymphocytes in the uterine epithelium as the placenta developed. This was tested by determining whether or not a reduction of lymphocytes occurred as attachment progressed. Lymphocytes were also counted during the oestrous cycle for comparative purposes.

\section{Materials and Methods}

Groups of 4-6 nulliparous heifers were slaughtered on Days 19, 21, 23, 25 and 27 after insemination and additional groups of 4 unmated heifers at 5, 10,15 and 20 days after oestrus. Animals were stunned using a captive bolt pistol and the reproductive tracts were removed immediately after exsanguination. Small volumes $(2-4 \mathrm{ml})$ of fixative, containing $1 \%(\mathrm{w} / \mathrm{v})$ paraformaldehyde, $3 \%$ $(\mathrm{v} / \mathrm{v})$ glutaraldehyde and $0.01 \%(\mathrm{w} / \mathrm{v})$ trinitroresorcinol in phosphate buffer $(0 \cdot 1 \mathrm{M}, \mathrm{pH} 7 \cdot 2)$, were 
injected into the chorioallantoic cavity of pregnant heifers to fix the embryonic membranes and maintain apposition. Each uterine horn was perfused via the middle uterine artery with $100 \mathrm{ml}$ fixative. Circular segments $2 \mathrm{~cm}$ in length were obtained from the oviducal, middle and body regions of both the ipsilateral and contralateral horns. Each segment was then divided and trimmed into 6 blocks, yielding a total of 36 specimens per animal. The blocks were immersed in fresh fixative for a minimum of $24 \mathrm{~h}$, dehydrated through an ascending alcohol series, infiltrated and embedded in glycol methacrylate (Sorvall JB-4 embedding medium), sectioned at $2 \mu \mathrm{m}$ and stained with haematoxylin and eosin.

Lymphocyte counts. The numbers of lymphocytes were counted in a $5 \mathrm{~mm}$ length of uterine epithelium that was orientated with the plane of section passing vertically through the epithelium. Initially, low power photographs $(\times 78)$ were taken and a montage made for each slide to define the $5 \mathrm{~mm}$ lengths ( 36 montages per animal). The $5 \mathrm{~mm}$ lengths were then examined at higher magnification $(\times 400-1000)$ and only those cells that could positively be identified as lymphocytes were counted. Subsequently, to reduce expense and time, a simplified method was implemented. This involved projecting a low-powered magnification of the section image $(\times 75)$ through a $48 \mathrm{~cm}$ television monitor and tracing the epithelial surface onto a sheet of semitransparent drafting paper mounted over the video screen. This latter method was compared to the original montages and both gave comparable results for determination of the $5 \mathrm{~mm}$ lengths.

The original objective was to count lymphocytes in the caruncular and intercaruncular regions. However, only a very small proportion of the endometrium is formed into caruncles during early gestation in heifers so $5 \mathrm{~mm}$ segments were not available in any of the animals. Thus, the results pertain almost entirely to intercaruncular tissue.

Statistical analysis. Lymphocyte data for pregnant and cyclic heifers were analysed by a splitplot analysis of variance using a Statistical Analysis System program (Helwig \& Council, 1979). Date, the whole plot factor, was compared to animal within date. The sub-plot factor, uterine location, was compared to animal by location within dates. Comparisons of individual means were done using least significant difference when $F$ values were significant. Since these were count data, a test for homogeneity of variance was made by comparing the ratio of $\mathrm{s}^{2} \mathrm{max} / \mathrm{s}^{2} \mathrm{~min}$ for each group of animals. One heifer in the Day 25 and one in the Day 27 group were eliminated from the analysis on the basis of a maximum normed residual test (Snedecor \& Cochran, 1980). Whenever an estimate of variance is given it represents the standard error of the mean.

\section{Results}

\section{Two unusual animals}

The data obtained from one animal slaughtered on Day 25 and another on Day 27 after insemination indicated a dramatically higher lymphocyte population in the uterine epithelium than means recorded in other pregnant heifers. The lymphocyte numbers in the ipsilateral horns were very similar to other Day 25 or 27 animals but numbers within the contralateral horns were much higher. These animals had extremely large total and within animal variance and were outside the $5 \%$ limit when tested by the normed residual test.

The above heifers exhibited an unusual biological situation because the chorion had not extended beyond the uterine body and into the contralateral horn. The maternal epithelial layers in the ipsilateral horns were very similar to the other animals in their respective groups with regions of squamous maternal epithelium plus binucleate and multinucleate cells interspaced with areas of columnar to cuboidal cells. In contrast, the contralateral horn contained no chorion and the epithelium was composed entirely of columnar cells. Therefore, since these two animals were statistically and biologically different from other heifers in their groups, they were excluded from the statistical analysis. 


\section{Qualitative observations}

Lymphocytes, with dense oval or slightly irregular nuclei surrounded by a thin zone of clear basophilic cytoplasm, were present in the basal, middle and apical regions of the uterine epithelium of unmated heifers (Pl. 1, Fig. 1). Lymphocytes of similar appearance and location were also found in the contralateral horn of the 2 pregnant animals with no chorionic membranes on that side (P1. 2, Fig. 4). The numbers and location of intraepithelial lymphocytes in females killed on Days 19 and 21 of gestation were generally similar to those observed in the cyclic animals.

The chorion was preserved in contact with the uterine epithelium in the middle region of the ipsilateral horn on Day 19 and in the ipsilateral middle and body regions at Day 21. Chorionic tissue was present in the contralateral oviducal segments in 4 of 5 heifers slaughtered on Day 21 . Attachment spread from the ipsilateral middle and body regions so that contact was maintained throughout both horns after Day 23.

The attachment was uniformly diffuse throughout the middle and body regions at Days 25 and 27 but only occasionally preserved in the oviducal segments. Intraepithelial lymphocyte numbers decreased (Text-fig. 1) and degenerate cells in the uterine epithelium increased as gestation progressed. Some of the degenerate cells were epithelial while others, with small pycnotic nuclei and scant cytoplasm, were presumably lymphocytes (PI. 2, Fig. 5). Between Days 23 and 27, aggregates of pycnotic nuclei and cellular debris were occasionally found between the chorion and maternal epithelium (Pl. 2, Fig. 6) or at the mouths of some endometrial glands (Pl. 2, Fig. 7). These were most common at Day 25 and probably formed through clumping of degenerate cells extruded from the epithelial surface or through the glandular lumina.

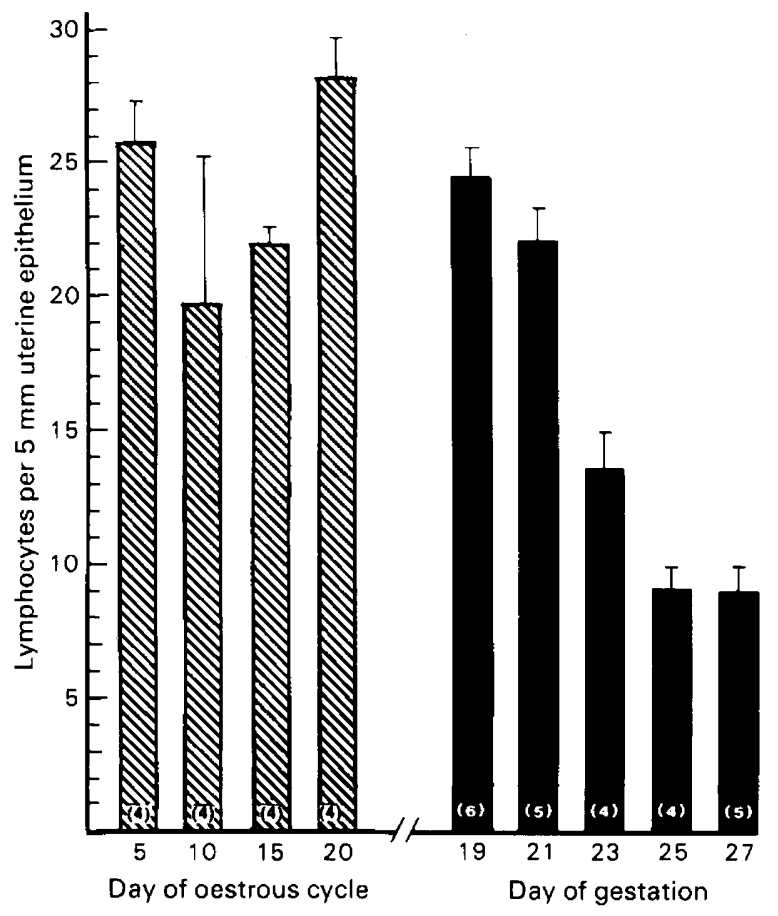

Text-fig. 1. Mean \pm s.e.m. number of intraepithelial lymphocytes per $5 \mathrm{~mm}$ of bovine uterine epithelium during the oestrous cycle and early gestation. Figures in parentheses indicate number of animals contributing to each mean, with 6 replicates from 6 uterine locations per animal. 
Globule leucocytes within and accumulations of eosinophils beneath the maternal epithelium were occasionally observed in pregnant and cyclic heifers. The globule leucocytes were quite rare and numbers did not increase as lymphocytes were reduced.

The maternal epithelium in the middle region of the ipsilateral horn changed substantially as attachment progressed. This resulted from invasion by chorionic binucleated cells and formation of multinucleated cells, accompanied by some degeneration of the maternal epithelium and flattening of multinucleates into syncytium. On Day 23, many regions of the maternal epithelium were still columnar with lymphocytes present (P1. 1, Fig. 2), while adjacent portions displayed predominantly multinucleate cells and syncytium (Pl. 1, Fig. 3). Lymphocytes were no longer found when the maternal side of the interface was composed of syncytium.

Table 1. Mean \pm s.e.m. intraepithelial lymphocytes per $5 \mathrm{~mm}$ of bovine uterine endometrium at different locations during early gestation

\begin{tabular}{|c|c|c|c|c|c|c|c|c|}
\hline \multirow{2}{*}{$\begin{array}{l}\text { Day of } \\
\text { gestation }\end{array}$} & \multirow{2}{*}{$\begin{array}{l}\text { No. of } \\
\text { heifers }\end{array}$} & \multicolumn{3}{|c|}{ Ipsilateral horn } & \multicolumn{3}{|c|}{ Contralateral horn } & \multirow{2}{*}{$\begin{array}{c}\text { Overall } \\
\text { mean } \pm \text { s.e.m }\end{array}$} \\
\hline & & Oviducal & Middle & Body & Body & Middle & Oviducal & \\
\hline 19 & 6 & $17 \cdot 5 \pm 1 \cdot 0$ & $25 \cdot 0 \pm 3 \cdot 1$ & $25 \cdot 5 \pm 2 \cdot 2$ & $26 \cdot 6 \pm 2 \cdot 9$ & $30 \cdot 0 \pm 2 \cdot 4$ & $22 \cdot 4 \pm 2 \cdot 2$ & $24 \cdot 5 \pm 1 \cdot 1^{a}$ \\
\hline 21 & 5 & $18 \cdot 0 \pm 0.7$ & $19.5 \pm 1.8$ & $25 \cdot 2 \pm 4 \cdot 2$ & $24 \cdot 7 \pm 4 \cdot 4$ & $22 \cdot 9 \pm 1 \cdot 1$ & $22 \cdot 1 \pm 3 \cdot 1$ & $22 \cdot 1 \pm 1 \cdot 2^{4}$ \\
\hline 23 & 4 & $12.3 \pm 0.9$ & $13 \cdot 3 \pm 3 \cdot 5$ & $10.9 \pm 1.9$ & $13.9 \pm 5 \cdot 0$ & $14.8 \pm 5 \cdot 1$ & $16 \cdot 5 \pm 2.6$ & $13 \cdot 6 \pm 1 \cdot 3^{\mathrm{ab}}$ \\
\hline 25 & 4 & $12 \cdot 3 \pm 1 \cdot 0$ & $6 \cdot 3 \pm 1 \cdot 4$ & $7 \cdot 1 \pm 0.8$ & $8.9 \pm 1.6$ & $5.9 \pm 1.5$ & $14 \cdot 5 \pm 1 \cdot 0$ & $9 \cdot 1 \pm 0.8^{\mathrm{b}}$ \\
\hline 27 & 5 & $13 \cdot 3 \pm 2 \cdot 7$ & $4 \cdot 2 \pm 0 \cdot 8$ & $7.9 \pm 1.5$ & $7 \cdot 0 \pm 1 \cdot 5$ & $8 \cdot 6 \pm 1 \cdot 3$ & $12 \cdot 9 \pm 1 \cdot 7$ & $9 \cdot 0 \pm 0 \cdot 9^{b}$ \\
\hline \multicolumn{2}{|c|}{ Overall } & $15 \cdot 0 \pm 0 \cdot 8^{a b}$ & $14 \cdot 5 \pm 2 \cdot 0^{\mathrm{a}}$ & $16 \cdot 3 \pm 2 \cdot 1^{\mathrm{abc}}$ & $17 \cdot 1 \pm 2 \cdot 2^{\mathrm{abc}}$ & $17 \cdot 5 \pm 2 \cdot 2^{b c}$ & $18 \cdot 1 \pm 1 \cdot 3^{c}$ & \\
\hline
\end{tabular}

Pooled data means (vertical) and location means (horizontal) with same superscript are not significantly different, $P>0.05$.

\section{PLATE 1}

All sections were from the intercaruncular regions of heifer uteri, embedded in methacrylate; H \& E stain.

Fig. 1. Intraepithelial lymphocytes $(\boldsymbol{\Delta})$ located at and away from the basal lamina. Ipsilateral horn, middle region, Day 20 after oestrus.

Fig. 2. Chorion attached to columnar uterine epithelium with intraepithelial lymphocytes $(\boldsymbol{\Delta})$ located at the basal lamina. Ipsilateral horn, middle region, Day 23 of gestation.

Fig. 3. Chorion attached to the maternal epithelium composed of multinucleate cells and syncytium. Note absence of intraepithelial lymphocytes in this figure which was taken from the same section as Fig. 2.

\section{PLATE 2}

Fig. 4. Columnar uterine epithelium with intraepithelial lymphocytes (A) present at various levels. Section from the utero-tubal region of the contralateral horn from an unusual animal with no chorionic vesicle extension into this horn, Day 25 of gestation.

Fig. 5. Intraepithelial lymphocytes $(\mathbf{\Delta})$ beside a binucleate cell. These lymphocytes are possibly migrating towards the interface and may subsequently become incorporated into a degenerate clump as illustrated in Fig. 6. Ipsilateral horn, middle region, Day 23 of gestation.

Fig. 6. Accumulation of pycnotic nuclei and degenerating cytoplasm between the chorion and maternal epithelium. These probably form through clumping of degenerate epithelial cells and lymphocytes. Ipsilateral horn, middle region, Day 25 of gestation.

Fig. 7. Accumulation of pycnotic nuclei at the mouth of a uterine gland. Ipsilateral horn, middle region, Day 25 of gestation. 
PLATE 1

PLATF 2
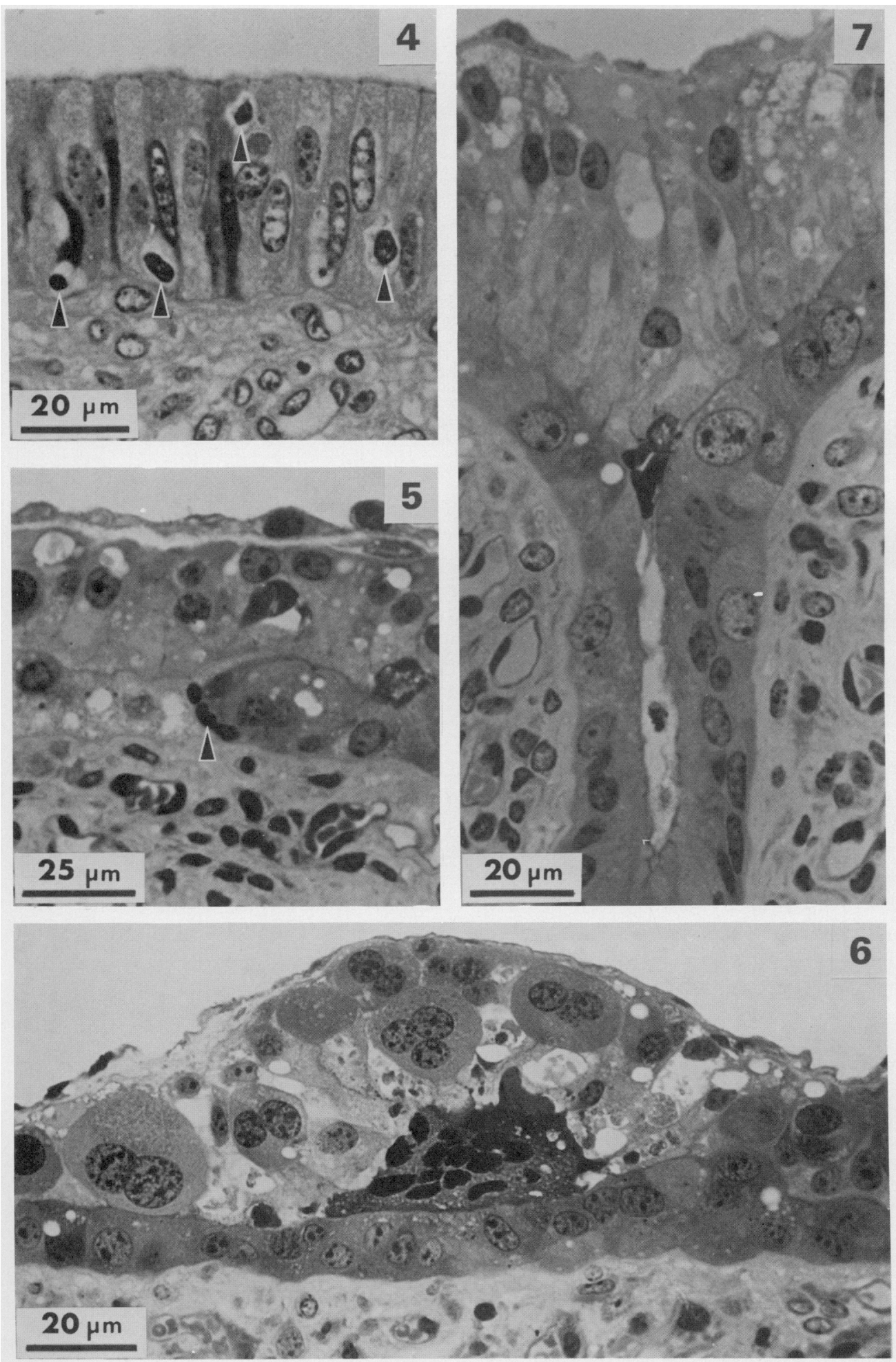


\section{Quantitative results}

In the cyclic animals, mean lymphocyte numbers per $5 \mathrm{~mm}$ of epithelium were $25.8 \pm 1.5$ on Day $5,19 \cdot 7 \pm 5 \cdot 5$ on Day $10,22 \cdot 0 \pm 0 \cdot 6$ on Day 15 and $28 \cdot 2 \pm 1 \cdot 5$ on Day 20 after oestrus (Text-fig. 1). Animals at Day 19 and 21 of gestation had similar lymphocyte numbers with means of $24.5 \pm$ $1 \cdot 1$ and $22 \cdot 1 \pm 1 \cdot 2$ respectively. As gestation progressed, the lymphocyte numbers decreased to means of $9.2 \pm 0.8$ on Day 25 and $9.0 \pm 0.9$ on Day 27 (Text-fig. 1). Data analysis for numbers of lymphocytes in cyclic animals indicated no significant difference between dates $(P>0.01)$. In early pregnant animals significant differences were found between dates $(P<0.005)$, locations $(P$ $<0.05)$ and date by location interaction $(P<0.005)$. The significant interaction resulted because, even though lymphocyte numbers were reduced in all segments over time, the oviducal regions had the lowest lymphocyte population at Day 19 but the greatest numbers at Day 27 (Table 1).

As attachment was consolidated, chorionic binucleated cells migrated across the placental interface and the maternal epithelium changed from columnar to squamous and syncytial. This flattening of the epithelium resulted in a reduction in the total number of nuclei per $5 \mathrm{~mm}$. Throughout the period studied, this change was mostly confined to the ipsilateral middle segment. The total number of nuclei were counted in 6 randomly selected $5 \mathrm{~mm}$ sections of the ipsilateral middle region on each of Days 19 and 27 of gestation: the numbers of nuclei per $5 \mathrm{~mm}$ were $730 \pm$ 42 on Day 19 and $280 \pm 40$ on Day 27 for a ratio of $2 \cdot 7: 1$. At this location the ratio of Day 19 to Day 27 lymphocytes per $5 \mathrm{~mm}$ was $6: 1$ (Table 1). Lymphocytes were therefore reduced per unit length and also in relation to the number of epithelial nuclei. Most epithelium in other regions remained columnar and the number of nuclei per $5 \mathrm{~mm}$ did not change.

Day 21-23 appears to be the critical period for lymphocyte changes in the uterine epithelium since a substantial reduction and the greatest variability were recorded at this time (Table 1).

\section{Discussion}

Lymphocyte numbers in the uterine epithelium did not change significantly throughout the oestrous cycle in this or the one previous study in which numbers were counted and analysed (Skjerven, 1956). Mean lymphocytes observed at the end of the 3rd week of gestation were similar to values throughout the cycle. As gestation progressed, numbers were systematically reduced to substantially lower means by Days 25 and 27 . Attachment begins in the middle segment of the ipsilateral horn on Day 19 and spreads throughout most of the uterine lumen during the next week (King et al., 1982). As attachment is consolidated, chorionic binucleated cells migrate across the fetal-maternal interface and fuse eventually to form the syncytium (Wooding, 1982). The flattening of multinucleated cells into syncytium results in a reduction of epithelial nuclei per unit length. This was the major reason for counting lymphocytes over distance rather than in proportion to the number of epithelial nuclei.

When the mean changes in lymphocyte numbers for all locations on Days 19 and 27 are compared, it appears that the decline is similar to the decrease in epithelial cells. However, epithelial cell counts were conducted only in the middle region of the ipsilateral horn where the greatest remodelling of the maternal surface was occurring. A closer examination of the individual lymphocyte counts in this location (Table 1) revealed that the ratio of Day 19 to 27 lymphocyte numbers in the ipsilateral middle regions was $6: 1$, whereas the change in epithelial cell numbers was only $2 \cdot 7: 1$.

A slight reduction in lymphocyte numbers was observed in the ipsilateral middle segment between Days 19 and 21. Thereafter, lymphocyte numbers decreased in all segments in a pattern that generally parallels binucleated cell migration and the spreading of attachment. The chorionic binucleated cells, involved with syncytial formation, have a paternal genetic component and, if paternal antigens were expressed, should be attractive to maternal immunocompetent cells. 
However, an immunological protection role for the syncytium has been proposed (Davies \& Wimsatt, 1966) and lymphocytes were not found in association with syncytial areas. Since very little syncytial tissue was seen before Day 23, no substantial reduction in lymphocytes had yet occurred. Syncytial tissue was never seen in the contralateral tips and only rarely in the ipsilateral tips. These observations suggest the presence of less intimate attachment and possibly a reduced immunosuppressive influence in these regions, leading to the higher lymphocyte populations recorded at Days 25 and 27.

Aggregates of pycnotic nuclei were observed between the chorion and maternal epithelium in the majority of animals slaughtered on Day 25 and occasionally in heifers killed on Days 23 and 27. These probably resulted from desquamated maternal epithelium plus some degenerate lymphocytes. Once the placenta is established, fewer lymphocytes may reach the epithelium and migration might be restricted to regions where syncytium has not formed. Lymphocytes could also be re-routed through the glandular epithelium to produce the clumps found at gland mouths. Day 18 bovine embryos produce a factor that inhibits lymphocyte transformation in vitro (French \& Northey, 1983) and later conceptuses might produce a cytotoxic factor which affects those lymphocytes that continue to migrate towards the placenta. These may then clump to form masses of degenerate cells at or near the gland openings. The bovine chorion actively engulfs and digests degenerating maternal cells during this stage of gestation (Wathes \& Wooding, 1980; King et al., 1981, 1982) and the clumps would represent one component of the histotroph.

The two heifers eliminated from the data analysis for statistical and biological reasons, one on Day 25 and another on Day 27, perhaps provided some of the most conclusive evidence in support of the original hypothesis that lymphocyte numbers in the uterine epithelium would be reduced as attachment developed. In both of these animals the presence of the growing embryo in the ipsilateral horn was associated with low lymphocyte numbers. In contrast, the opposite horn, which contained no chorion, had high numbers of intraepithelial lymphocytes and counts often exceeded those obtained in cyclic animals.

The potentially antigenic chorion attaching to and becoming incorporated into the maternal epithelium did not attract additional lymphocytes. By the end of the 4th week of gestation, the number of lymphocytes per unit length of uterine epithelium was significantly lower than the numbers present on Days 19 and 21 when attachment began. The results suggest that the conceptus has some ability to mask its presence from and perhaps reduce the migration of maternal lymphocytes towards the placental interface.

Financial support was provided by the Natural Sciences and Engineering Research Council of Canada and the Ontario Ministry of Agriculture and Food.

\section{References}

Davies, J. \& Winsatt, W.A. (1966) Observations on the fine structure of the sheep placenta. Acta anat. 65, 182-223.

French, L.R. \& Northey, D.L. (1983) Inhibitory effect of bovine conceptus on lymphocyte stimulation. $J$. Anim. Sci. 57, 456-465.

Helwig, J.T. \& Council, K.A. (1979) SAS user's guide. SAS Institute Inc., Raleigh.

King, G.J., Atkinson, B.A. \& Robertson, H.A. (1981) Development of the intercaruncular areas during early gestation and establishment of the bovine placenta. J. Reprod. Fert. 61, 469-474.

King, G.J., Atkinson, B.A. \& Robertson, H.A. (1982) Implantation and early placentation in domestic ungulates. J. Reprod. Fert., Suppl. 31, 17-30.
Skjerven, O. (1956) Endometrial biopsy studies in reproductively normal cattle. Acta endocr., Copenh., Suppl. 26, 1-101.

Snedecor, G.W. \& Cochran, W.G. (1980) Statistical Methods. Iowa State University Press, Ames.

Wathes, D.C. \& Wooding, F.B.P. (1980) An electron microscopic study of implantation in the cow. Am.J. Anat. 159, 285-306.

Wille, K.H. \& Leiser, R. (1977) Adenosine triphosphatases in the bovine endometrium and in the trophoblast during implantation. Microscopic and electron microscopic investigations. Anat. Anz. 141, 401-419.

Wooding, F.B.P. (1982) The role of the binucleate cell in ruminant placental structure. J. Reprod. Fert., Suppl. 31, 31-39. 